\title{
Sensitivity of the Moment of Inertia of Neutron Stars to the Equation of State of Neutron-Rich Matter
}

\author{
F. J. Fattoyev ${ }^{1, \text { * }}$ and J. Piekarewicz ${ }^{1, \dagger}$ \\ ${ }^{1}$ Department of Physics, Florida State University, Tallahassee, Florida 32306, USA
}

(Dated: November 11, 2018)

\begin{abstract}
The sensitivity of the stellar moment of inertia to the neutron-star matter equation of state is examined using accurately-calibrated relativistic mean-field models. We probe this sensitivity by tuning both the density dependence of the symmetry energy and the high density component of the equation of state, properties that are at present poorly constrained by existing laboratory data. Particularly attractive is the study of the fraction of the moment of inertia contained in the solid crust. Analytic treatments of the crustal moment of inertia reveal a high sensitivity to the transition pressure at the core-crust interface. This may suggest the existence of a strong correlation between the density dependence of the symmetry energy and the crustal moment of inertia. However, no correlation was found. We conclude that constraining the density dependence of the symmetry energy - through, for example, the measurement of the neutron skin thickness in ${ }^{208} \mathrm{~Pb}$ - will place no significant bound on either the transition pressure or the crustal moment of inertia.
\end{abstract}

PACS numbers: 26.60.-c,26.60.Kp,21.60.Jz

*Electronic address: ff07@fsu.edu

${ }^{\dagger}$ Electronic address: jpiekarewicz@fsu.edu 


\section{INTRODUCTION}

Since their discovery more than 40 years ago, neutron stars have been a fruitful testing ground for both general relativity and theories of dense matter. Being extremely compact (with typical compactness parameters of $R_{\mathrm{s}} / R \equiv 2 G M / c^{2} R \sim 0.4$ ) and extremely dense (with densities exceeding several times nuclear matter saturation density) neutron stars are unique laboratories for the study of exotic phenomena that lie well outside the realm of terrestrial laboratories. Moreover, neutron stars provide a natural meeting place for nuclear physics and astrophysics. And with the advent and commissioning of powerful facilities dedicated to the production of rare isotopes, new telescopes operating at a variety of wavelengths, and more sensitive gravitational wave detectors, the partnership between nuclear physics and astrophysics will only continue to grow.

In the present contribution we study the sensitivity of the stellar moment of inertia to the underlying equation of state. Although the formalism is general - at least within the slow-rotation approximation - we focus on the recently discovered binary pulsar PSR J07373039 [1, 2]. Located ten times closer than the celebrated Hulse-Taylor binary system [3] and with the shortest orbital period of its kind (almost three times smaller than the HulseTaylor binary), PSR J0737-3039 is the first ever discovered double pulsar. This discovery has resulted in some of the most precise tests of Einstein's general theory of relativity to date. Moreover, it has also enabled the accurate determination of several pulsar properties, such as the orbital period of the binary, both pulsar masses, and both spin periods. However, their individual radii, moments of inertia, gravitational redshifts, or any other property that would allow a simultaneous mass-radius determination - and therefore place important constraints on the equation of state - are still unavailable. Yet the prospects for measuring the moment of inertia of the fastest spinning pulsar in the binary (PSR J0737-3039A) have never been better. Doing so with even a $10 \%$ accuracy may provide stringent constraints on the nuclear equations of state [4 7]. Note, however, that the proposed 10\% accuracy has been recently put into question [8]. Yet we trust that the various observational challenges will be met successfully in the near future. With such a view in mind, we focus on a particular feature of the equation of state (EOS) that has a strong impact on the moment of inertia: the nuclear symmetry energy.

The nuclear symmetry energy represents the increase in the energy of the system as protons are converted into neutrons (or neutrons into protons). Whereas ground-state masses constrain the symmetry energy near saturation density, they leave its density dependence (slope, curvature, etc.) largely undetermined. This is important as the slope of the symmetry energy at saturation density determines the pressure of pure neutron matter (PNM). And it is precisely the pressure of PNM that provides the necessary stellar support against gravitational collapse. Thus, the larger the pressure of PNM the larger the radius of the neutron star. Note that it is also the pressure of PNM that accounts for the size of the neutron skin thickness in medium to heavy nuclei. As a result, a strong correlation was uncover between the neutron skin thickness of ${ }^{208} \mathrm{~Pb}$ and the neutron radius of a neutron star [9]. Given that the moment of inertia scales as the square of the stellar radius, the aim of this work is to study the expected correlation between the neutron skin thickness of

${ }^{208} \mathrm{~Pb}$ and the stellar moment of inertia. We should mention that at the time of this writing the experimental effort to measure the neutron radius of ${ }^{208} \mathrm{~Pb}$ at the Jefferson Laboratory was well on its way. The Parity Radius Experiment ("PREx") at the Jefferson Laboratory aims to measure the neutron radius of ${ }^{208} \mathrm{~Pb}$ accurately and model independently via parity- 
violating electron scattering [10, 11]. PREx should provide a unique experimental constraint on the neutron skin thickness of a heavy nucleus and correspondingly on the pressure of pure neutron matter. We should also mention that considerable progress has been achieved in the theoretical front. By building on the universal behavior of dilute Fermi gases with an infinite scattering length, significant theoretical progress has been made in constraining the equation of state of pure neutron matter [12 21].

While the overall moment of inertia of the neutron star is of great interest, the fraction of the moment of inertia contained in the stellar crust may be as useful in constraining the equation of state [22, 23]. Indeed, by studying the sudden and fairly regular spin jumps ("pulsar glitches") in the Vela pulsar, it was determined that at least $1.4 \%$ of the total moment of inertia of the star must reside in the solid crust [22]. Pulsar glitches are believed to be the result of angular momentum transfer between the star's solid crust and a more rapidly rotating superfluid component residing in the stellar interior. As the crustal moment of inertia is sensitive to the transition pressure at the core-crust interface [22], the above ( $\gtrsim 1.4 \%$ ) limit may place a significant constraint on the EOS of dilute, neutron-rich matter. In this way, the crustal moment of inertia may provide an attractive astrophysical complement to PREx and to the dynamics of dilute Fermi gases in constraining the density dependence of the symmetry energy.

The paper has been organized as follows. We start Sec. II by providing the background material necessary to compute the moment of inertia of a neutron star in the slow-rotation approximation [24, 25]. Later on in the section we introduce the various accurately-calibrated relativistic mean-field models that will be used to compute the equation of state of the stellar material [26 29]. We note that whereas all the models reproduce various experimentally measured properties of finite nuclei, they differ significantly in their predictions for the equation of state at both low and high densities. In Sec. III we present results for various neutron-star properties and explore their possible correlation to the neutron skin thickness of ${ }^{208} \mathrm{~Pb}$. Finally, Sec. IV contains our concluding remarks.

\section{FORMALISM}

In this section we develop the formalism required to compute the stellar moment of inertia. The section consists of two main components: (a) the equations of stellar structure and (b) the neutron-star matter equation of state.

\section{A. Equations of stellar structure}

The cornerstone of our approach is the slow-rotation approximation pioneered by Hartle and Thorne [24, 25]. We assume that the neutron star is rotating uniformly with a stellar frequency $\Omega$ that is far smaller than the Kepler frequency at the equator. That is,

$$
\Omega \ll \Omega_{\max } \approx \sqrt{\frac{G M}{R^{3}}} .
$$

The formalism - even in the slow-rotation approximation - is subtle, primarily due to the dragging of local inertial frames. The expression for the moment of inertia of an axisymmetric star in hydrostatic equilibrium is derived in great detail in Refs. [30, 31, so we only 
summarize here some of the most important results. For a more pedagogical discussion one may consult the text by Glendenning [32].

In the slow-rotation (first order in the angular velocity) approximation the moment of inertia of a uniformly rotating, axially symmetric neutron star is given by the following expression:

$$
I \equiv \frac{J}{\Omega}=\frac{8 \pi}{3} \int_{0}^{R} r^{4} e^{-\nu(r)} \frac{\bar{\omega}(r)}{\Omega} \frac{(\mathcal{E}(r)+P(r))}{\sqrt{1-2 G M(r) / r}} d r
$$

where $J$ is the angular momentum, $\nu(r)$ and $\bar{\omega}(r)$ are radially-dependent metric functions (see below) and $M(r), \mathcal{E}(r)$, and $P(r)$ are the stellar mass, energy density, and pressure profiles, respectively. Perhaps the greatest advantage of the slow-rotation approximation is that all the quantities appearing in Eq. (2) may be assumed to remain spherically symmetric. This implies that all stellar profiles may be determined from the Tolman-OppenheimerVolkoff (TOV) equation. That is,

$$
\begin{aligned}
& \frac{d P(r)}{d r}=-G \frac{(\mathcal{E}(r)+P(r))\left(M(r)+4 \pi r^{3} P(r)\right)}{r^{2}(1-2 G M(r) / r)} \\
& \frac{d M(r)}{d r}=4 \pi r^{2} \mathcal{E}(r) .
\end{aligned}
$$

Given boundary conditions in terms of a central pressure $P(0)=P_{c}$ and $M(0)=0$, the TOV equations may be solved once an equation of state $(P=P(\mathcal{E}))$ is supplied. In particular, the stellar radius $R$ and mass $M$ are determined from the following two conditions: $P(R)=0$ and $M=M(R)$.

Once mass and pressure profiles have been obtained, the full space-time metric may be determined. We note that in the slow-rotation approximation the invariant interval for the background metric of a stationary, axially symmetric star may be written as [24, 33]:

$$
d s^{2}=g_{\mu \nu} d x^{\mu} d x^{\nu}=-e^{2 \nu(r)} d t^{2}+e^{2 \lambda(r)} d r^{2}+r^{2} d \theta^{2}+r^{2} \sin ^{2} \theta d \phi^{2}-2 \omega(r) r^{2} \sin ^{2} \theta d t d \phi .
$$

As alluded earlier, in the slow-rotation approximation the spherically symmetric metric functions $\nu(r)$ and $\lambda(r)$ remain unchanged from their values obtained in the limit of a nonrotating, static, and spherically symmetric neutron star. In particular, $\lambda(r)$ is simply related to the mass profile $M(r)$ by

$$
g_{11}(r)=e^{2 \lambda(r)}=(1-2 G M(r) / r)^{-1} .
$$

Further, $\nu(r)$ can be determined from solving a first-order differential equation or, equivalently, from evaluating the following integral:

$$
\nu(r)=\frac{1}{2} \ln \left(1-\frac{2 G M}{R}\right)-G \int_{r}^{R} \frac{\left(M(x)+4 \pi x^{3} P(x)\right)}{x^{2}(1-2 G M(x) / x)} d x
$$

Finally, one must determine the metric function $\omega(r)$ - the one new ingredient that emerges from the slow rotation and which has no counterpart in Newtonian gravity. The frequency $\omega(r)$ appears as a consequence of the dragging of local inertial frames by the rotating star; 
the so-called Lense-Thirring effect. The effective (or relative) frequency $\bar{\omega}(r) \equiv \Omega-\omega(r)$ appearing in Eq. (2) represents the angular velocity of the fluid as measured in a local inertial reference frame. In particular, the dimensionless relative frequency $\widetilde{\omega}(r) \equiv \bar{\omega}(r) / \Omega$ satisfies the following second-order differential equation:

$$
\frac{d}{d r}\left(r^{4} j(r) \frac{d \widetilde{\omega}(r)}{d r}\right)+4 r^{3} \frac{d j(r)}{d r} \widetilde{\omega}(r)=0
$$

where

$$
j(r)=e^{-\nu(r)-\lambda(r)}= \begin{cases}e^{-\nu(r)} \sqrt{1-2 G M(r) / r} & \text { if } r \leq R, \\ 1 & \text { if } r>R .\end{cases}
$$

Note that $\widetilde{\omega}(r)$ is subject to the following two boundary conditions:

$$
\begin{aligned}
& \widetilde{\omega}^{\prime}(0)=0, \\
& \widetilde{\omega}(R)+\frac{R}{3} \widetilde{\omega}^{\prime}(R)=1 .
\end{aligned}
$$

Also note that in the slow-rotation approximation the moment of inertia does not depend on the stellar frequency $\Omega$. In practice, one chooses an arbitrary value for the central frequency $\widetilde{\omega}_{c}=\widetilde{\omega}(0)$ and numerically integrates Eq. (7) up to the edge of the star. In general, the boundary condition at the surface [Eq. (9b)] will not be satisfied for an arbitrary choice of $\widetilde{\omega}_{c}$, so one must rescale the function and its derivative by an appropriate constant to correct for the mismatch.

The procedure described above provides all the necessary steps to compute the integrand in Eq. (2). The moment of inertia is then obtained by performing the one remaining integral using standard numerical techniques. Having solved for both $\widetilde{\omega}(r)$ and $I$, one could check the consistency of the formalism by ensuring that the following equation is satisfied:

$$
\widetilde{\omega}^{\prime}(R)=\frac{6 G I}{R^{4}}
$$

\section{B. The crustal moment of inertia}

It has been suggested that pulsar glitches, namely, the sudden increase in the spin rate of pulsars, may place important constraints on the equation of state [22, 34]. In particular, an analysis based on a long time observation of glitches of the Vela pulsar suggests that at least $1.4 \%$ of the total moment of inertia must reside in the non-uniform crust [22, 34]. This is interesting as the crustal moment of inertia is particularly sensitive to the transition pressure at the core-crust interface and this observable is believed to be correlated to the densitydependence of the symmetry energy [35 38]. Thus, it is both interesting and enlightening to obtain analytic expressions for the crustal moment of inertia.

The crustal moment of inertia is defined in terms of the general expression provided in Eq. (2) but with the range of the integral now limited from the transition (or core) radius $R_{t}$ to the stellar radius $R$. That is,

$$
I_{c r}=\frac{8 \pi}{3} \int_{R_{t}}^{R} r^{4} e^{-\nu(r)} \widetilde{\omega}(r) \frac{(\mathcal{E}(r)+P(r))}{\sqrt{1-2 G M(r) / r}} d r .
$$


However, given that the crust is thin and the density within it is low, several approximation have been proposed that help render the integral tractable [22, 23, 34, 39, 40]. The various approximations and details on how to evaluate the integral are left to the appendix. In particular, we show that under those approximations the TOV equation may be solved exactly. Here, however, we simply quote our final result:

$$
I_{c r} \approx \frac{16 \pi}{3} \frac{R_{t}^{6} P_{t}}{R_{s}}\left[1-\left(\frac{R_{s}}{R}\right)\left(\frac{I}{M R^{2}}\right)\right]\left[1+\frac{48}{5}\left(R_{t} / R_{s}-1\right)\left(P_{t} / \mathcal{E}_{t}\right)+\ldots\right],
$$

where $R_{s}=2 G M$ is the Schwarzschild radius of the star, and $P_{t}=P\left(R_{t}\right)$ and $\mathcal{E}_{t}=\mathcal{E}\left(R_{t}\right)$ are the pressure and energy density at the core-crust interface. The ellipsis in the above equation indicates that the derivation was carried out to first order in the small quantity $P_{t} / \mathcal{E}_{t} \lesssim 0.01$. A few comments are in order. First, the above expression for the crustal moment of inertia is extremely accurate (of the order of a few percent; see Tables IV and V]. Second, the two terms in brackets in Eq. 12 provide each a moderate $\sim 10 \%$ correction to the leading term, with the corrections being of opposite sign. Third, although the crustal moment of inertia still depends on the total moment of inertia $I$, one may preserve the accuracy of our result without having to rely on a highly accurate estimate of $I$. In particular, by using the simple relationship proposed in Ref. [40], namely,

$$
\frac{I}{M R^{2}}=\frac{0.21}{1-R_{s} / R}
$$

one obtains

$$
I_{c r} \approx \frac{16 \pi}{3} \frac{R_{t}^{6} P_{t}}{R_{s}}\left[1-\frac{0.21}{\left(R / R_{s}-1\right)}\right]\left[1+\frac{48}{5}\left(R_{t} / R_{s}-1\right)\left(P_{t} / \mathcal{E}_{t}\right)+\ldots\right] .
$$

This expression remains extremely accurate, yet has the added appeal that for a neutron star with a given mass $M$ (or $R_{s}$ ) and radius $R$, the crustal moment of inertia depends exclusively on $R_{t}, P_{t}$, and $\mathcal{E}_{t}$ - all quantities that are expected to be sensitive to the density dependence of the symmetry energy. Note that the approximation for the total moment of inertia given in Eq. (13) has been put into question in Ref. 34]. However, for our purposes such an approximation is adequate as $I$ enters into the expression for the crustal moment of inertia as a small correction. No such approximation will be made for $I$ when reporting the fractional moment of inertia $I_{c r} / I$. Indeed, no approximation for $I$ will be made at all.

For completeness, we include an expression for the crustal mass that was derived in the appendix following similar steps. We obtain,

$$
M_{c r} \approx 8 \pi R_{t}^{3} P_{t}\left(R_{t} / R_{s}-1\right)\left[1+\frac{32}{5}\left(R_{t} / R_{s}-3 / 4\right)\left(P_{t} / \mathcal{E}_{t}\right)+\ldots\right]
$$

\section{Neutron-star matter equation of state}

As alluded earlier, the structure of neutron stars is sensitive to the equation of state of cold, fully catalyzed, neutron-rich matter. Spanning many orders of magnitude in density, neutron stars display rich and exotic phases that await a detailed theoretical understanding. For example, at densities that are about one third of nuclear matter saturation density and 
below, the uniform ground state becomes unstable against clustering correlations. This nonuniform region constitutes the stellar crust, which itself is divided into an inner and an outer region. In the outer crust the system is organized into a Coulomb lattice of neutron-rich nuclei embedded in a degenerate electron gas [41,42]. For this region we employ the equation of state of Baym, Pethick, and Sutherland [41]. With increasing density the nuclei become progressively more neutron rich until the neutron drip region is reached; this region defines the boundary between the outer and the inner crust. It has been speculated that the bottom layers of the inner crust consist of complex and exotic structures that are collectively known as nuclear pasta [39, 43, 44]. Whereas significant progress has been made in simulating this exotic region [45-47], a detailed equation of state is still missing. Hence, we resort to a fairly accurate polytropic EOS to interpolate between the outer solid crust and the uniform liquid interior [22, 48]. For the uniform liquid core - with densities as low as one-third and as high as ten times nuclear-matter saturation density - we generate the equation of state using various refinements to the relativistic mean-field model of Serot and Walecka [49 51. For consistency, the same models are used to compute the transition density from the liquid core to the solid crust. This we do by searching for the critical density at which the uniform system becomes unstable to small amplitude density oscillations. The stability analysis of the uniform ground state is based on a relativistic random-phase-approximation (RPA) as detailed in Ref. [48.

The equation of state for the uniform liquid core is based on an interacting Lagrangian that has been accurately calibrated to a variety of ground-state properties of both finite nuclei and infinite nuclear matter. The model includes a nucleon field $(\psi)$, two isoscalar mesons (a scalar $\phi$ and a vector $V^{\mu}$ ), and one isovector meson $\left(b^{\mu}\right)$ [50, 51] (the photon field plays no role in the present discussion of infinite nuclear matter at the mean-field level). The free Lagrangian density for this model is given by

$$
\begin{aligned}
\mathscr{L}_{0} & =\bar{\psi}\left(i \gamma^{\mu} \partial_{\mu}-M\right) \psi+\frac{1}{2} \partial_{\mu} \phi \partial^{\mu} \phi-\frac{1}{2} m_{s}^{2} \phi^{2} \\
& -\frac{1}{4} F^{\mu \nu} F_{\mu \nu}+\frac{1}{2} m_{v}^{2} V^{\mu} V_{\mu}-\frac{1}{4} \mathbf{b}^{\mu \nu} \mathbf{b}_{\mu \nu}+\frac{1}{2} m_{\rho}^{2} \mathbf{b}^{\mu} \mathbf{b}_{\mu},
\end{aligned}
$$

where $F_{\mu \nu}$ and $\mathbf{b}_{\mu \nu}$ are the isoscalar and isovector field tensors, respectively. That is,

$$
\begin{aligned}
& F_{\mu \nu}=\partial_{\mu} V_{\nu}-\partial_{\nu} V_{\mu}, \\
& \mathbf{b}_{\mu \nu}=\partial_{\mu} \mathbf{b}_{\nu}-\partial_{\nu} \mathbf{b}_{\mu} .
\end{aligned}
$$

Further, the parameters $M, m_{s}, m_{v}$, and $m_{\rho}$ represent the nucleon and meson masses and may be treated (if wished) as empirical constants. The interacting Lagrangian density is given by the following expression [26, 50, 51]:

$$
\mathscr{L}_{\text {int }}=\bar{\psi}\left[g_{\mathrm{s}} \phi-\left(g_{\mathrm{v}} V_{\mu}+\frac{g_{\rho}}{2} \boldsymbol{\tau} \cdot \mathbf{b}_{\mu}\right) \gamma^{\mu}\right] \psi-U\left(\phi, V^{\mu}, \mathbf{b}^{\mu}\right) .
$$

The model includes Yukawa couplings $\left(g_{\mathrm{s}}, g_{\mathrm{v}}\right.$, and $\left.g_{\rho}\right)$ between the nucleon and the three meson fields. However, to improve the phenomenological standing of the model - for example, to soften the compressibility of symmetric nuclear matter - the Lagrangian density must be supplemented by nonlinear meson interactions. These are given by

$$
U\left(\phi, V^{\mu}, \mathbf{b}^{\mu}\right)=\frac{\kappa}{3 !}\left(g_{\mathrm{s}} \phi\right)^{3}+\frac{\lambda}{4 !}\left(g_{\mathrm{s}} \phi\right)^{4}-\frac{\zeta}{4 !}\left(g_{\mathrm{v}}^{2} V_{\mu} V^{\mu}\right)^{2}-\Lambda_{\mathrm{v}}\left(g_{\rho}^{2} \mathbf{b}_{\mu} \cdot \mathbf{b}^{\mu}\right)\left(g_{\mathrm{v}}^{2} V_{\nu} V^{\nu}\right) .
$$


Details on the calibration procedure may be found in Refs. [50 53] and references therein. Note that additional local terms of the same order in a power-counting scheme could have been included. However, their phenomenological impact has been shown to be small [26], so they have not been included in the calibration procedure. Of particular interest and of critical important to the present study is the isoscalar-isovector coupling term $\Lambda_{\mathrm{v}}[52$, 53]. Such a term has been added to the Lagrangian density to modify the poorly known density dependence of the symmetry energy - a property that is predicted to be stiff (i.e., to increase rapidly with density) in most relativistic mean-field models. The addition of $\Lambda_{\mathrm{v}}$ provides a simple - yet efficient and reliable - method of tuning the density dependence of the symmetry energy without sacrificing the success of the model in reproducing experimentally constrained ground-state observables. Because of the sensitivity of the stellar radius to the density dependence of the symmetry energy [9], we expect a strong correlation between $\Lambda_{\mathrm{v}}$ and the stellar moment of inertia.

Whereas the full complexity of the quantum system can not be tackled, the ground-state properties of the system may be computed in a mean-field (MF) approximation. In the MF approximation all the meson fields are replaced by their classical expectation values and their solution can be readily obtained by solving the classical Euler-Lagrange equations of motion. The sole remnant of quantum behavior is in the treatment of the nucleon field which emerges from a solution to the Dirac equation in the presence of appropriate scalar and vector potentials [50, 51]. Following standard mean-field practices, the energy density of the system is given by the following expression:

$$
\mathcal{E}=\frac{1}{\pi^{2}} \int_{0}^{k_{\mathrm{F}}^{p}} k^{2} E_{k}^{*} d k+\frac{1}{\pi^{2}} \int_{0}^{k_{\mathrm{F}}^{n}} k^{2} E_{k}^{*} d k+g_{\mathrm{v}} V_{0} \rho_{\mathrm{B}}+\frac{g_{\rho}}{2} b_{0} \rho_{3}+U\left(\phi_{0}, V_{0}, b_{0}\right),
$$

where $E_{k}^{*}=\sqrt{k^{2}+M^{* 2}}, M^{*}=M-g_{\mathrm{s}} \phi_{0}$ is the effective nucleon mass, $k_{\mathrm{F}}^{p}\left(k_{\mathrm{F}}^{n}\right)$ is the proton (neutron) Fermi momentum, $\rho_{\mathrm{B}}\left(\rho_{3}\right)$ is the isoscalar (isovector) baryon density, and $U\left(\phi_{0}, V_{0}, b_{0}\right)$ is given by the following expression:

$$
\begin{aligned}
U\left(\phi_{0}, V_{0}, \mathbf{b}_{0}\right) & =\frac{1}{2} m_{s}^{2} \phi_{0}^{2}+\frac{\kappa}{3 !}\left(g_{\mathrm{s}} \phi_{0}\right)^{3}+\frac{\lambda}{4 !}\left(g_{\mathrm{s}} \phi_{0}\right)^{4} \\
& -\frac{1}{2} m_{v}^{2} V_{0}^{2}-\frac{\zeta}{4 !}\left(g_{\mathrm{v}} V_{0}\right)^{4}-\frac{1}{2} m_{\rho}^{2} b_{0}^{2}-\Lambda_{\mathrm{v}}\left(g_{\mathrm{v}} V_{0}\right)^{2}\left(g_{\rho} b_{0}\right)^{2} .
\end{aligned}
$$

The expression for the energy density may be "simplified" by using the classical equations of motion for the vector fields to express the isoscalar and isovector densities $\rho_{\mathrm{B}}$ and $\rho_{3}$ in terms of $V_{0}$ and $b_{0}$. One obtains,

$$
\begin{aligned}
\mathcal{E} & =\frac{1}{\pi^{2}} \int_{0}^{k_{\mathrm{F}}^{p}} k^{2} E_{k}^{*} d k+\frac{1}{\pi^{2}} \int_{0}^{k_{\mathrm{F}}^{n}} k^{2} E_{k}^{*} d k \\
& +\frac{1}{2} m_{s}^{2} \phi_{0}^{2}+\frac{\kappa}{3 !}\left(g_{\mathrm{s}} \phi_{0}\right)^{3}+\frac{\lambda}{4 !}\left(g_{\mathrm{s}} \phi_{0}\right)^{4} \\
& +\frac{1}{2} m_{v}^{2} V_{0}^{2}+\frac{\zeta}{8}\left(g_{\mathrm{v}} V_{0}\right)^{4}+\frac{1}{2} m_{\rho}^{2} b_{0}^{2}+3 \Lambda_{\mathrm{v}}\left(g_{\mathrm{v}} V_{0}\right)^{2}\left(g_{\rho} b_{0}\right)^{2}
\end{aligned}
$$

Finally, as the MF approximation is thermodynamically consistent, the pressure of the system (at zero temperature) may be obtained either from the energy-momentum tensor or from the energy density and its first derivative with respect to the density [50, 51]. That is,

$$
P=\frac{1}{3 \pi^{2}} \int_{0}^{k_{\mathrm{F}}^{p}} \frac{k^{4}}{E_{k}^{*}} d k+\frac{1}{3 \pi^{2}} \int_{0}^{k_{\mathrm{F}}^{n}} \frac{k^{4}}{E_{k}^{*}} d k-U\left(\phi_{0}, V_{0}, b_{0}\right)
$$




\begin{tabular}{|l||c|c|c|c|c|c|c|c|c|c|}
\hline Model & $m_{\mathrm{s}}$ & $m_{\mathrm{v}}$ & $m_{\rho}$ & $g_{\mathrm{s}}^{2}$ & $g_{\mathrm{v}}^{2}$ & $g_{\rho}^{2}$ & $\kappa$ & $\lambda$ & $\zeta$ & $\Lambda_{\mathrm{v}}$ \\
\hline \hline NL3 & 508.194 & 782.501 & 763.000 & 104.3871 & 165.5854 & 79.6000 & 3.8599 & -0.01591 & 0.00 & 0.00 \\
MS & 485.000 & 782.500 & 763.000 & 111.0428 & 216.8998 & 70.5941 & 0.5083 & +0.02772 & 0.06 & 0.00 \\
FSUGold & 491.500 & 782.500 & 763.000 & 112.1996 & 204.5469 & 138.4701 & 1.4203 & +0.02376 & 0.06 & 0.03 \\
\hline
\end{tabular}

TABLE I: Parameter sets (coupling constants and masses) for the mean-field models used in the text. The parameter $\kappa$ and the meson masses $m_{\mathrm{s}}, m_{\mathrm{v}}$, and $m_{\rho}$ are all given in $\mathrm{MeV}$. The nucleon mass has been fixed at $M=939 \mathrm{MeV}$ in all the models.

Given that neutron-star matter is fully catalyzed, chemical equilibrium must be imposed. Namely, the composition of the star is determined from the equality of the chemical potential of the various species. That is,

$$
\mu_{n}=\mu_{p}+\mu_{e}=\mu_{p}+\mu_{\mu}
$$

Note that electrons and muons are assumed to behave as relativistic free Fermi gases (with $\left.m_{e} \equiv 0\right)$. Of course, muons appear in the system only after the electronic Fermi momentum becomes equal to the muon rest mass. The total energy density and pressure of the star are simply obtained by adding up the nucleonic and leptonic contributions.

\section{RESULTS}

To study the sensitivity of the stellar moment of inertia to the equation of state we will use relativistic mean-field models that have been accurately calibrated to the properties of infinite-nuclear matter (MS) [26], to the ground-state properties of finite nuclei (NL3) [27, 28], or to both (FSUGold) [29]. Parameter sets for these three models are listed in Table I.

The predicted equations of state - pressure vs energy density - for the three models are displayed in Fig. 1. Given that the equation of state for the solid crust is identical in all three models, we only present the contribution from the uniform liquid core. The lowest energy density and pressure depicted in the figure - which are different in all three modelssignal the transition from the uniform liquid core to the non-uniform solid crust. Note that the uniform core is assumed to consist of nucleons and leptons (electrons and muons) in chemical equilibrium; no exotic degrees of freedom are considered.

The resulting equations of state show a significant model dependence. As the models have been accurately calibrated, this is a clear indication that the existing database of nuclear observables is insensitive to both the low- and high-density behavior of the EOS (the energy density at saturation is about $140 \mathrm{MeV} / \mathrm{fm}^{3}$ ). In this contribution we are particularly interested in the sensitivity of the stellar moment of inertia to the two empirical parameters $\zeta$ and $\Lambda_{\mathrm{v}}$-with the former controlling the high-density behavior of the EOS and the latter the density dependence of the symmetry energy.

The NL3 parameter set [27, 28] provides an excellent description of the ground-state properties of finite nuclei (such as masses and charge radii) without invoking either $\zeta$ or $\Lambda_{\mathrm{v}}$ (see Table I). As a consequence of having set $\zeta=\Lambda_{\mathrm{v}} \equiv 0$, NL3 generates a fairly stiff equation of state. At sub-saturation density, this behavior is reflected in the relatively small value of the energy density at the core-crust interface. At the high-density extreme, NL3 approaches the stiffest possible equation of state that is consistent with causality (i.e., $P=\mathcal{E}$ ). As we 


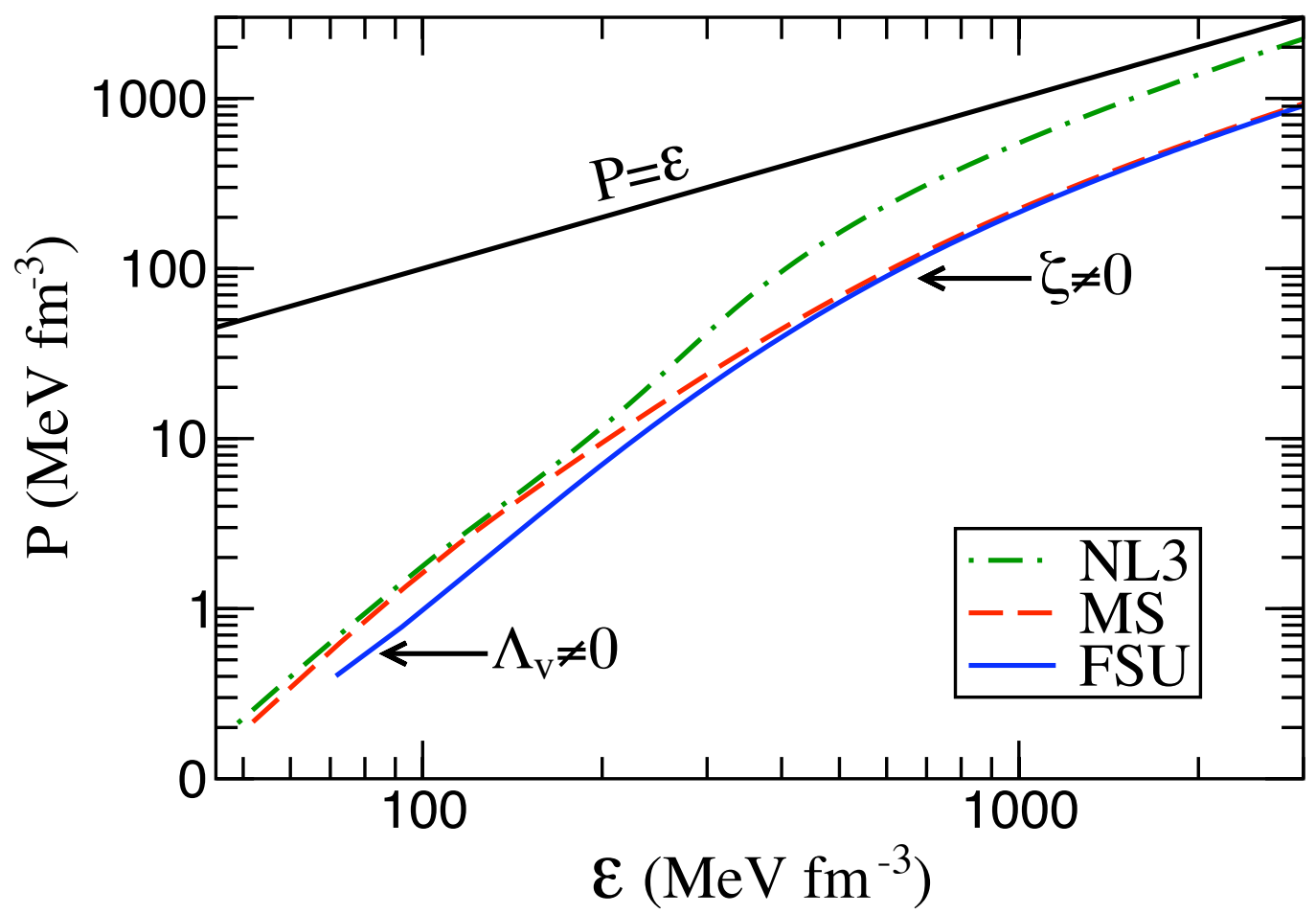

FIG. 1: (Color online) Equation of state (pressure vs energy density) of neutron-star matter predicted by the three relativistic mean-field models discussed in the text. The solid black line $(P=\mathcal{E})$ denotes the stiffest possible equation of state consistent with causality.

shall see below, such a stiff EOS generates neutron stars that are both massive and large (see Fig. 2 and Table II).

As far as the MS equation of state is concerned, Müller and Serot were able to build models with a wide range of values for $\zeta$ that while reproducing the same observed properties at normal nuclear densities, they produce maximum neutron star masses that differ by almost one solar mass [26]. By selecting a value of $\zeta=0.06$, the MS model adopted here predicts a softer EOS and consequently a limiting neutron-star mass that is significantly smaller than NL3. Such a softening at high density is clearly evident in Fig. 1. Note that the $\zeta=0.06$ choice appears consistent with the dense-matter equation of state extracted from an analysis of energetic heavy-ion collisions [54, 55]. However, extracting the zero-temperature EOS from energetic heavy-ion collisions may be model dependent. Thus, observational data on neutron-star masses may provide the cleanest constraint on the high-density component of the equation of state. Note, however, that since $\Lambda_{\mathrm{v}}$ remains equal to zero in this model, the energy density and pressure at the core-crust interface remain largely unchanged from their NL3 values (see Table II).

The FSUGold parameter set is characterized by having both $\zeta$ and $\Lambda_{\mathrm{v}}$ different from zero [29]. By adding $\Lambda_{\mathrm{v}}$ to the model one can soften the EOS - particularly the symmetry energy - at low to intermediate densities. This produces a shift of the core-crust transition energy density and pressure to higher values relative to both NL3 and MS. Moreover, such a softening generates neutron stars of relative small radii (see Fig. 2 and Table II). We note that the softening of the symmetry energy is required to describe the isoscalar monopole and isovector dipole modes in medium to heavy nuclei [56]. Further, this softening appears 


\begin{tabular}{|l||c|c|c|c|c|c|}
\hline Model & $M_{\max }\left(M_{\odot}\right)$ & $R_{1.4}(\mathrm{~km})$ & $\mathcal{E}_{1.4}\left(\mathrm{MeV} \mathrm{fm}^{-3}\right)$ & $\rho_{t}\left(\mathrm{fm}^{-3}\right)$ & $\mathcal{E}_{t}\left(\mathrm{MeV} \mathrm{fm}^{-3}\right)$ & $P_{t}\left(\mathrm{MeV} \mathrm{fm}^{-3}\right)$ \\
\hline \hline NL3 & 2.78 & 15.05 & 269.63 & 0.052 & 48.96 & 0.212 \\
MS & 1.81 & 13.78 & 430.81 & 0.055 & 51.91 & 0.216 \\
FSUGold & 1.72 & 12.66 & 536.91 & 0.076 & 71.53 & 0.402 \\
\hline
\end{tabular}

TABLE II: Predictions for the maximum neutron star mass and for the radius and central energy density of a $1.4 M_{\odot}$ neutron star in the three relativistic mean-field models discussed in the text. The last three quantities represent the transition density, energy density, and pressure at the core-crust interface.
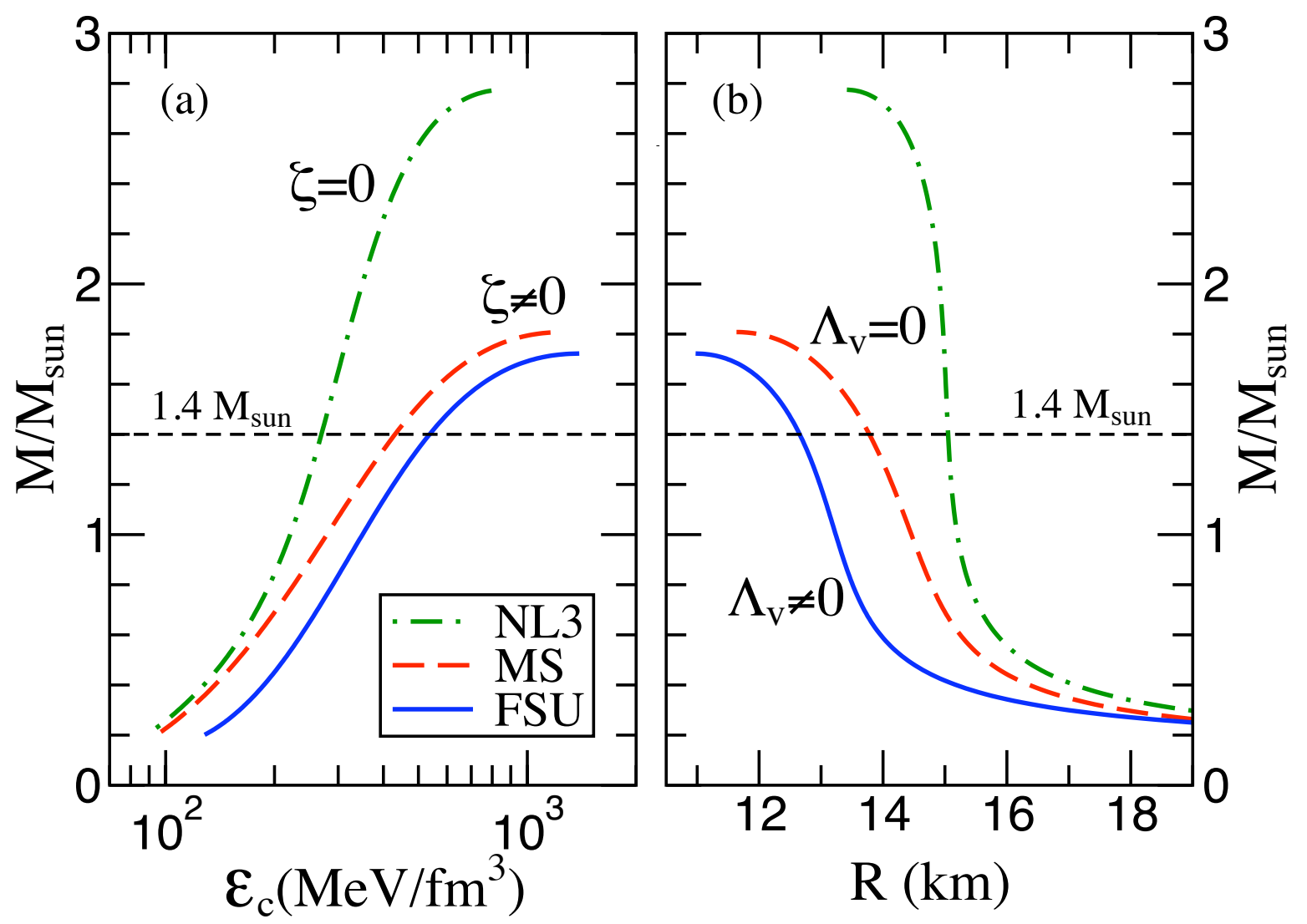

FIG. 2: (Color online) Neutron-star mass as a function of the central density (a) and the stellar radius (b) for the three relativistic mean-field models discussed in the text.

consistent with microscopic descriptions of the equation of state of low-density neutron matter (see Ref. [20] and references therein). On the other hand, the value of $\zeta=0.06$ adopted here is solely constrained by the dynamics of heavy ions. Yet, the reported errors are large enough to accommodate slightly stiffer equations of state (although not as stiff as NL3) [54, 55].

Having generated an equation of state, one can now proceed to solve the TOV equations [see Eqs. (3)]. Once a value for the central energy density $\mathcal{E}_{c}$ (or pressure $P_{c}$ ) is specified, solutions to the TOV equations are obtained in the form of mass $M(r)$, pressure $P(r)$, and energy density $\mathcal{E}(r)$ profiles. From these, the stellar radius $R$ is extracted from the pressure profile as the point at which the pressure vanishes, namely, $P(R)=0$. Similarly, the total 


\begin{tabular}{|l||c|c|c|c|c|c|c|}
\hline Model & $\Lambda_{\mathrm{v}}$ & $g_{\rho}^{2}$ & $L(\mathrm{MeV})$ & $R_{n}-R_{p}(\mathrm{fm})$ & $\rho_{t}\left(\mathrm{fm}^{-3}\right)$ & $\mathcal{E}_{t}\left(\mathrm{MeV} \mathrm{fm}^{-3}\right)$ & $P_{t}\left(\mathrm{MeV} \mathrm{fm}^{-3}\right)$ \\
\hline \hline NL3 & 0.00 & 79.6000 & 118.189 & 0.280 & 0.052 & 48.960 & 0.212 \\
& 0.01 & 90.9000 & 87.738 & 0.251 & 0.061 & 57.574 & 0.338 \\
& 0.02 & 106.0000 & 68.217 & 0.223 & 0.746 & 70.630 & 0.508 \\
& 0.03 & 127.1000 & 55.311 & 0.195 & 0.085 & 81.012 & 0.535 \\
& 0.04 & 158.6000 & 46.607 & 0.166 & 0.090 & 85.618 & 0.376 \\
\hline FSU & 0.00 & 80.2618 & 108.764 & 0.286 & 0.051 & 48.458 & 0.207 \\
& 0.01 & 93.3409 & 87.276 & 0.260 & 0.060 & 56.330 & 0.317 \\
& 0.02 & 111.5126 & 71.833 & 0.235 & 0.069 & 65.387 & 0.415 \\
& 0.03 & 138.4701 & 60.515 & 0.207 & 0.076 & 71.534 & 0.402 \\
& 0.04 & 182.6162 & 52.091 & 0.176 & 0.078 & 73.924 & 0.268 \\
& 0.05 & 268.0859 & 45.743 & 0.137 & 0.077 & 73.206 & 0.036 \\
\hline
\end{tabular}

TABLE III: The NL3 and FSUGold "families" of mean-field interactions. The isovector parameters $\Lambda_{\mathrm{v}}$ and $g_{\rho}$ were adjusted so that all models have a symmetry energy of $\approx 26 \mathrm{MeV}$ at a density of $\approx 0.1 \mathrm{fm}^{-3}$. The tuning of the isovector interaction modifies the slope of the symmetry energy $L$ at saturation density. The impact of such a change on the neutron skin thickness of ${ }^{208} \mathrm{~Pb}$ and on the transition density, energy density, and pressure at the core-crust interface are displayed in the last four columns.

stellar mass is obtained from the mass profile as $M=M(R)$. Note that for a given value of the central energy density $\mathcal{E}_{c}$, a unique point in the $M-R$ diagram is generated.

In Fig. 2 we display neutron-star masses as a function of the central energy density (left panel) and the stellar radius (right panel). The imprint of the underlying equation of state is clearly evident on these curves. For example, the stiff behavior of the NL3 equation of state is reflected on its very large limiting mass (of close to 3 solar masses). Also evident is the significant reduction in the maximum stellar mass as one softens the EOS by shifting the value of $\zeta$ from 0 to 0.06 . Finally, we observe a significant variation in the value of the central energy density required to produce a "canonical" $1.4 M_{\odot}$ neutron star. The NL3 equation of state is so stiff (i.e., the pressure gradient is so large) that a central energy density of only twice its value at saturation is sufficient to support the star against gravitational collapse. In contrast, the softer FSUGold equation of state requires a central energy density that is twice as large as NL3 or about 4 times its value at saturation.

Whereas $\zeta$ controls the maximum stellar mass, $\Lambda_{\mathrm{v}}$ controls the stellar radius. This is illustrated on the right-hand panel of Fig. 2. Although the MS and FSUGold equations of state display similar behavior at high density (see Fig. 1) the former generates stellar radii that are significantly larger than the latter. For example, for a $1.4 M_{\odot}$ neutron star the difference exceeds one kilometer. Note that the stellar radius - although primarily sensitive to the EOS at intermediate densities - is also sensitive to the high-density component of the EOS. Hence, although both NL3 and MS have $\Lambda_{\mathrm{v}}=0$, MS (with $\zeta \neq 0$ ) produces more compact stars.

Having established the critical role of the two empirical parameters $\zeta$ and $\Lambda_{\mathrm{v}}$ on the mass-vs-radius relationship of neutron stars, we now return to the central goal of the present manuscript: to assess the sensitivity of the stellar moment of inertia-both total and crustal - to the equation of state. To properly address this topic we must build models 


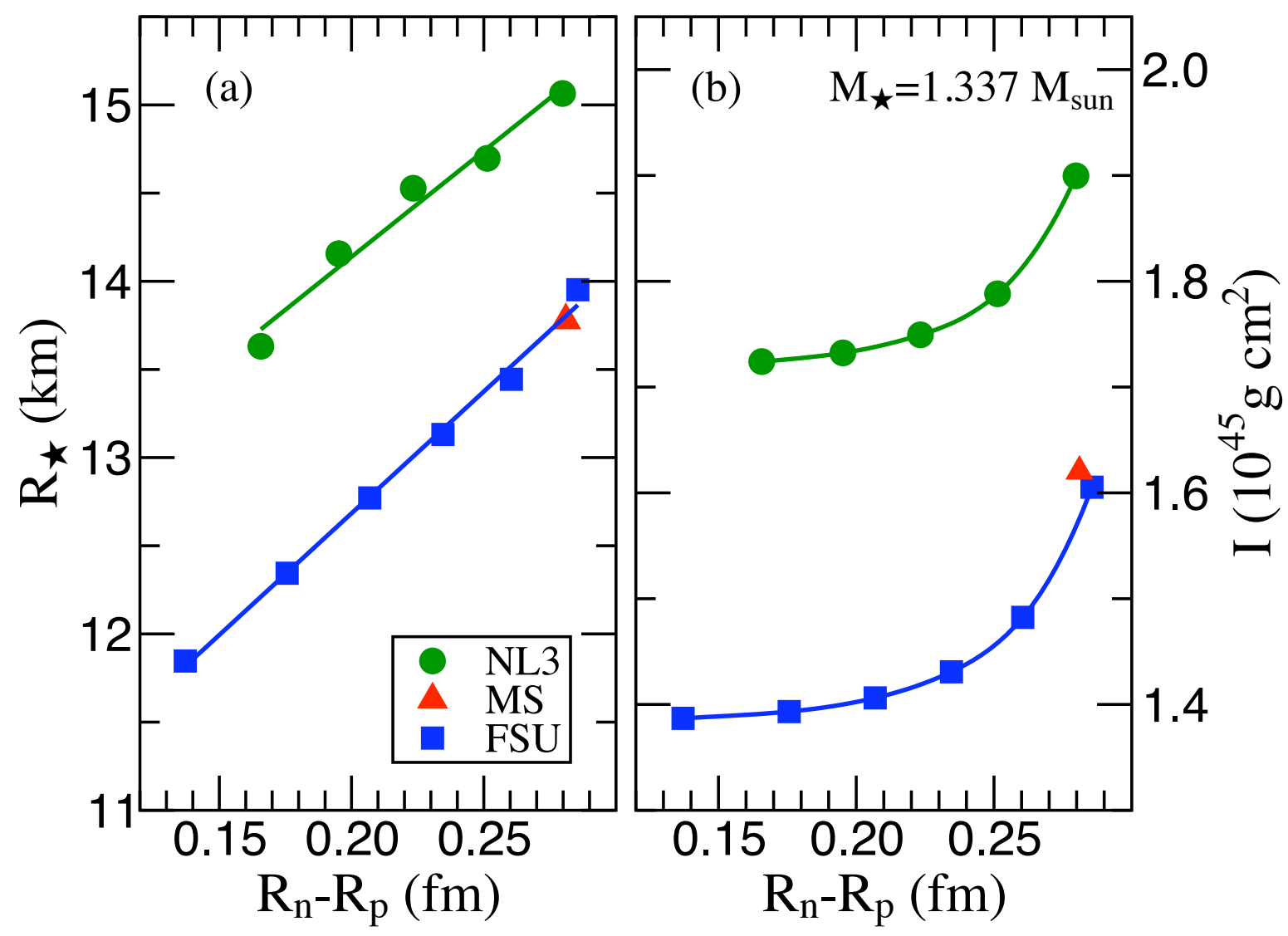

FIG. 3: (Color online) Stellar radius (a) and moment of inertia (b) of a $1.337 M_{\odot}$ neutron star (J0737-3039 A) as a function of the neutron skin thickness of ${ }^{208} \mathrm{~Pb}$.

that, while accurately calibrated, can generate a wide range of values for poorly constrained nuclear observables. To do so, we modify the density dependence of the symmetry energy by resorting to a simple - yet highly robust - procedure first introduced in Ref. [52]. The procedure consists on modifying the isovector mean-field interaction by simultaneously changing $\Lambda_{\mathrm{v}}$ and $g_{\rho}$ (the $N N \rho$ coupling constant) in such a way that the value of the symmetry energy remains fixed at a specific value of the baryon density. Given that nuclei have a low-density surface, the symmetry energy is best constrained not at nuclear matter saturation density, but at a slightly lower value [57]. In this contribution - as in Ref. [52] - we fixed the symmetry energy at $\approx 26 \mathrm{MeV}$ at a density of $\approx 0.1 \mathrm{fm}^{-3}$. This ensures that well constrained observables (such as masses and charge radii) remain consistent with their experimental values. Moreover, as this procedure involves the tuning of only the isovector interaction, all properties of symmetric nuclear matter remain intact. Yet poorly constrained observablessuch as the neutron skin thickness of heavy nuclei and neutron-star radii - can be made to vary over a wide range of values [9, 52]. In Table III we display the appropriate isovector parameters for the NL3 and FSUGold "families" of mean-field interactions. Particularly sensitive to this change is the slope of the symmetry energy $L$ at saturation density. The table illustrates the impact of $L$ on the neutron skin thickness of ${ }^{208} \mathrm{~Pb}$ and on the transition density, energy density, and pressure at the core-crust interface. Note that there is no need to generate an MS family given that it shares the same value of $\zeta$ with FSUGold. 
In Fig. 3 we display the radius and moment of inertia of a $M=1.337 M_{\odot}$ neutron star (such as J0737-3039 A) as a function of the neutron skin thickness in ${ }^{208} \mathrm{~Pb}$. Although many other observables (such as $L$ ) may be used to characterize the density dependence of the symmetry energy, we have selected the neutron-skin of ${ }^{208} \mathrm{~Pb}$ because it represents a fundamental nuclear observable that will soon be directly determined from laboratory data. The left-hand panel in the figure is reminiscent of the linear correlation between the neutron skin in ${ }^{208} \mathrm{~Pb}$ and the stellar radius uncovered in Ref. 9. Given that neutron stars and the neutron skin of heavy nuclei are both made of neutron-rich material, the emergence of such a correlation should not come as a surprise. However, knowledge of the neutron skin is not sufficient to determine the stellar radius. Whereas the neutron skin of heavy nuclei is sensitive to the EOS around saturation density, the stellar radius is also sensitive to its high-density component. Hence, to eliminate the model dependence one must rely on observational data rather than on laboratory experiments. The right-hand panel in Fig. 3 displays the corresponding moment of inertia. There is a significant drop in the moment of inertia as the neutron skin departs from its largest value (at $\Lambda_{\mathrm{v}}=0$ ) but then the sensitivity weakens. The same kind of behavior is displayed when $I$ is plotted as a function of the stellar radius (not shown). As in the case of the stellar radius, the total moment of inertia is sensitive to the high-density component of the EOS, so a strong model dependence remains. Note that since both MS and the stiffest member of the FSUGold family have $\Lambda_{\mathrm{v}}=0$ and $\zeta=0.06$, their predictions are very close to each other.

Given the sensitivity of the moment of inertia to the high density component of the EOS, we now shift our attention to its crustal component. Our expectation-based on its high sensitivity to the transition pressure [see Eq. (14)] - is that a strong correlation will emerge between the crustal moment of inertia $\left(I_{c r}\right)$ and the neutron skin thickness of ${ }^{208} \mathrm{~Pb}$. To our surprise, no such correlation exists. We attempt to elucidate this finding in what follows.

The core-crust boundary is determined by identifying the highest baryon density at which the uniform ground state becomes unstable against small amplitude density fluctuations. The stability analysis of the uniform ground state is based on a relativistic random-phase approximation (RPA) that is described in detail in Refs. [48, 52. As first proposed in Ref. [52] - and confirmed since using various equivalent approaches [21, 35, 37, 58] —a strong correlations emerges between the core-crust transition density $\left(\rho_{t}\right)$ and the neutron skin thickness of ${ }^{208} \mathrm{~Pb}$. The left-hand panel in Fig. 4 provides evidence for such a correlation. The figure displays the energy per nucleon as a function of baryon density for uniform, neutron-rich matter in chemical equilibrium. Results are displayed for the various members of the FSUGold family of mean-field interactions. The symbols in the figure are used to denote the transition point and are labeled according to the value of the neutron skin thickness in ${ }^{208} \mathrm{~Pb}$. The behavior displayed in Fig. 4(a) is simple to understand given the following facts: (a) all models predict identical properties for symmetric nuclear matter, (b) all models share the same value of the symmetry energy at $\approx 0.1 \mathrm{fm}^{-3}$, and (c) the softer the symmetry energy the smaller the neutron skin thickness in ${ }^{208} \mathrm{~Pb}$. Based on these fairly general assertions, one concludes that it is energetically expensive for the system to remain uniform if the equation of state is soft. Thus, models with thin neutron skins predict higher transition densities.

However, whereas a clear correlation emerges between the transition density and the neutron skin, no such correlations is observed in the case of the transition pressure. The right-hand panel in Fig. 4 displays the pressure of neutron-rich matter as a function of the baryon density. As expected, the larger the neutron skin thickness in ${ }^{208} \mathrm{~Pb}$ the larger the 


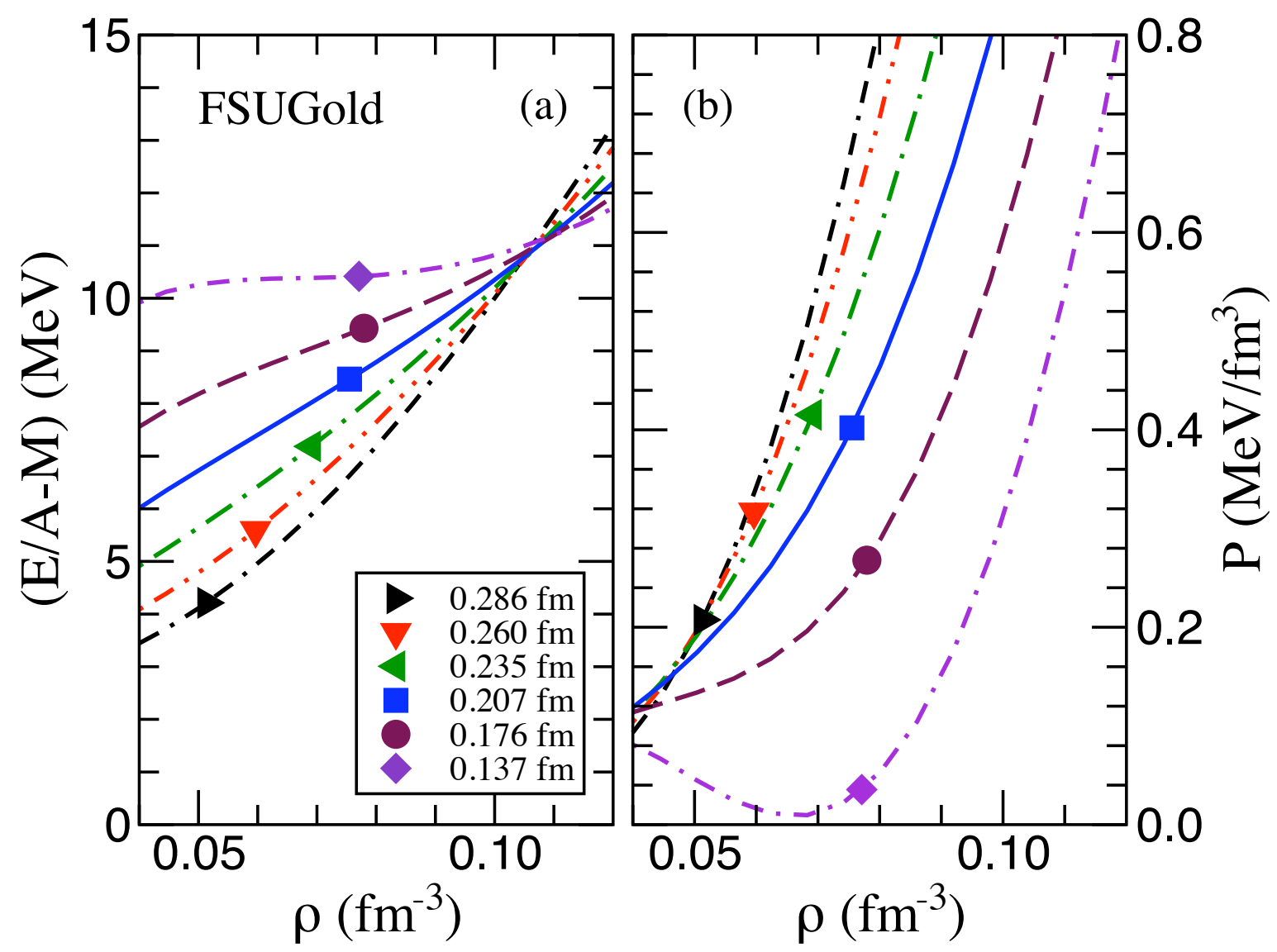

FIG. 4: (Color online) Equation of state for uniform, neutron-rich matter in beta equilibrium for the FSUGold family of mean-field interactions (labeled by the value of the neutron skin thickness in ${ }^{208} \mathrm{~Pb}$ ). The binding energy per nucleon (a) and the pressure (b) are displayed in parametric form in terms of the baryon density. The various symbols indicate the transition density, energy per nucleon, and pressure at which the uniform state becomes unstable against small density fluctuations.

pressure. That is, at a fixed given density, the pressure is larger for models that predict larger neutron skins. However, different models predict different transition densities and this mere fact destroys the correlation between the transition pressure and the neutron skin. For example, for the three stiffest models displayed in the figure, there is a direct correlation between the transition density and the transition pressure $\left(P_{t}\right)$. In this region these three models exhibit relatively little scatter so that an increase in $\rho_{t}$ is accompanied by a corresponding increase in $P_{t}$. However, as the models continue to soften, an inverse correlation develops: models with a soft symmetry energy have large transition densities but small transition pressures. As a result, no correlation between the transition pressure and the neutron skin thickness in ${ }^{208} \mathrm{~Pb}$ develops. Note that this result is consistent with the very recent analysis presented in Ref. [58].

We now proceed to examine the correlation between the neutron skin thickness of ${ }^{208} \mathrm{~Pb}$ and various quantities defined at the transition region. Before doing so, however, we display in Fig. 5 the energy density and pressure at the core-crust boundary as a function of the transition density. As suggested earlier, whereas the energy density is strongly correlated to the density at the interface, the pressure is not. In Fig. 6 we plot the value of various 


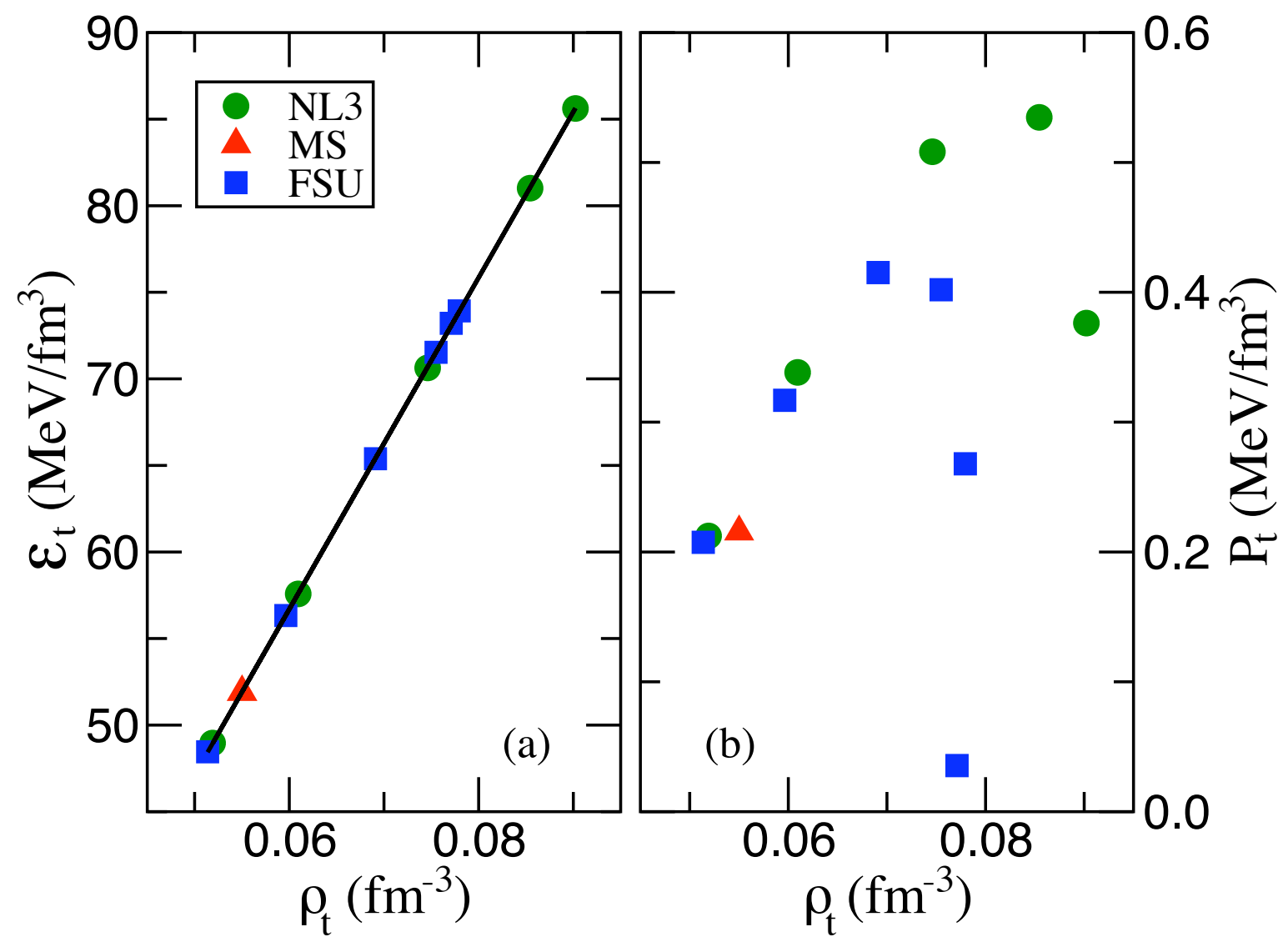

FIG. 5: (Color online) Properties of neutron-rich matter at the core-crust interface for the various models discussed in the text. Whereas the transition energy density is strongly correlated to the transition density (a), the transition pressure is not (b).

observables in the transition region as a function of the neutron skin thickness in ${ }^{208} \mathrm{~Pb}$. In addition to the transition density, energy density, and pressure, the proton fraction is also displayed. Excluding the softest model - which as we shall see below appears in conflict with an observational constraint - there is a clear (inverse) correlation between the transition density $\rho_{t}$ and $R_{n}-R_{p}$, as originally proposed in Ref. [52]. Not surprisingly [see Fig. 5(a)] the transition energy density displays an inverse correlation that is just as strong. In contrast, there is no correlation between the transition pressure and $R_{n}-R_{p}$. Indeed, one can find models that have neutron skins that vary by more than $0.1 \mathrm{fm}$ yet predict identical transition pressures. Finally, the proton fraction at the core-crust interface displays a tight inverse correlation. As mentioned earlier [see Fig 4(a)] it is energetically expensive for models with a soft-symmetry energy to support a large neutron-proton asymmetry. Thus, the thinner the neutron skin of ${ }^{208} \mathrm{~Pb}$, the larger the proton fraction.

Having explored the correlations (or lack-thereof) between the neutron skin thickness in ${ }^{208} \mathrm{~Pb}$ and various properties of the EOS in the transition region, we now proceed to study the sensitivity of several crustal properties to $R_{n}-R_{p}$. In particular, predictions for the crustal mass, thickness, and moment of inertia for a $1.337 M_{\odot}$ neutron star (such as J0737-3039 A) are displayed in Fig. 7. Note that approximate analytic expressions for the crustal moment of inertia $\left(I_{c r}\right)$ and mass $\left(M_{c r}\right)$ have been derived in the appendix and have been collected in Eqs. (14) and (15). Both of these expressions indicate a high sensitivity to 

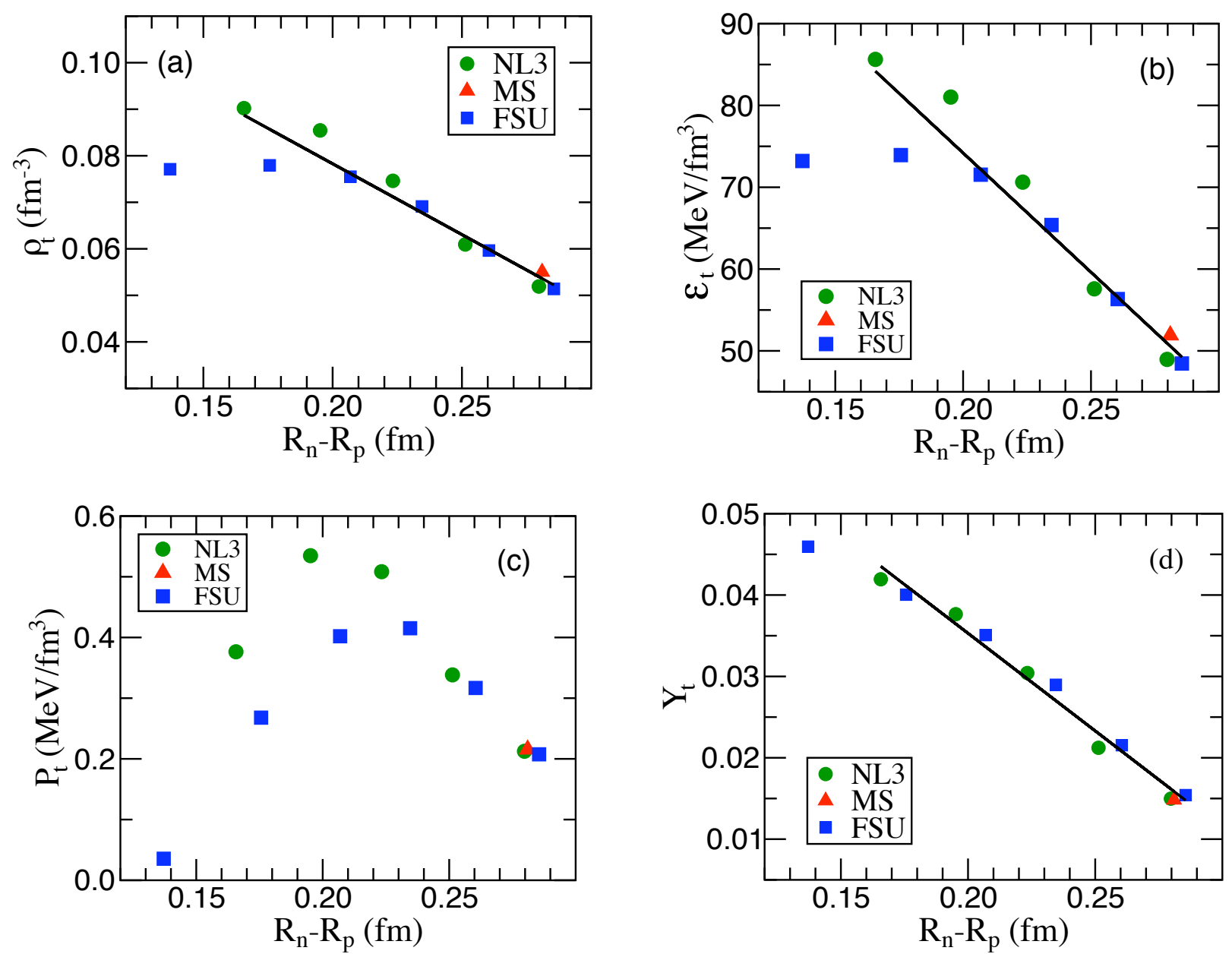

FIG. 6: (Color online) Baryon density (a), energy density (b), pressure (c), and proton fraction (d) at the core-crust interface as a function of the neutron skin thickness in ${ }^{208} \mathrm{~Pb}$ for the various mean-field interactions discussed in the text.

the transition pressure $P_{t}$. Indeed, the imprint of the transition pressure is clearly evident in all crustal observables. For example, given that for models with a soft symmetry energy the transition pressure increases with $R_{n}-R_{p}$, an initial (i.e., for small neutron skins) direct correlation develops between $R_{n}-R_{p}$ and all crustal properties. Eventually, however, the transition pressure reaches a maximum and then an inverse correlation ensues. Hence, we conclude that a measurement of the neutron skin thickness in ${ }^{208} \mathrm{~Pb}$ will place no significant constraint on the crustal mass, thickness, or moment of inertia. Note that the crustal thickness $R_{c r}$ also follows such a trend due to its dependence on the crustal mass; if a small crustal mass remains, then the crustal thickness will be small.

As mentioned in the Introduction, the study of pulsar glitches in the Vela pulsar suggests that at least $1.4 \%$ of the total moment of inertia must reside in the solid crust [22, 23]. The results displayed in Fig. 8 show how such a constrain may be used to rule out certain equations of state. The figure shows predictions for the fractional moment of inertia $\left(I_{c r}\right)$ of the binary pulsar J0737-3039 as a function of the neutron skin thickness in ${ }^{208} \mathrm{~Pb}$. We observe that the softest member of the FSUGold family predicts a fractional moment of inertia of only $0.35 \%$ - significantly lower than the $1.4 \%$ bound. This suggests that models 


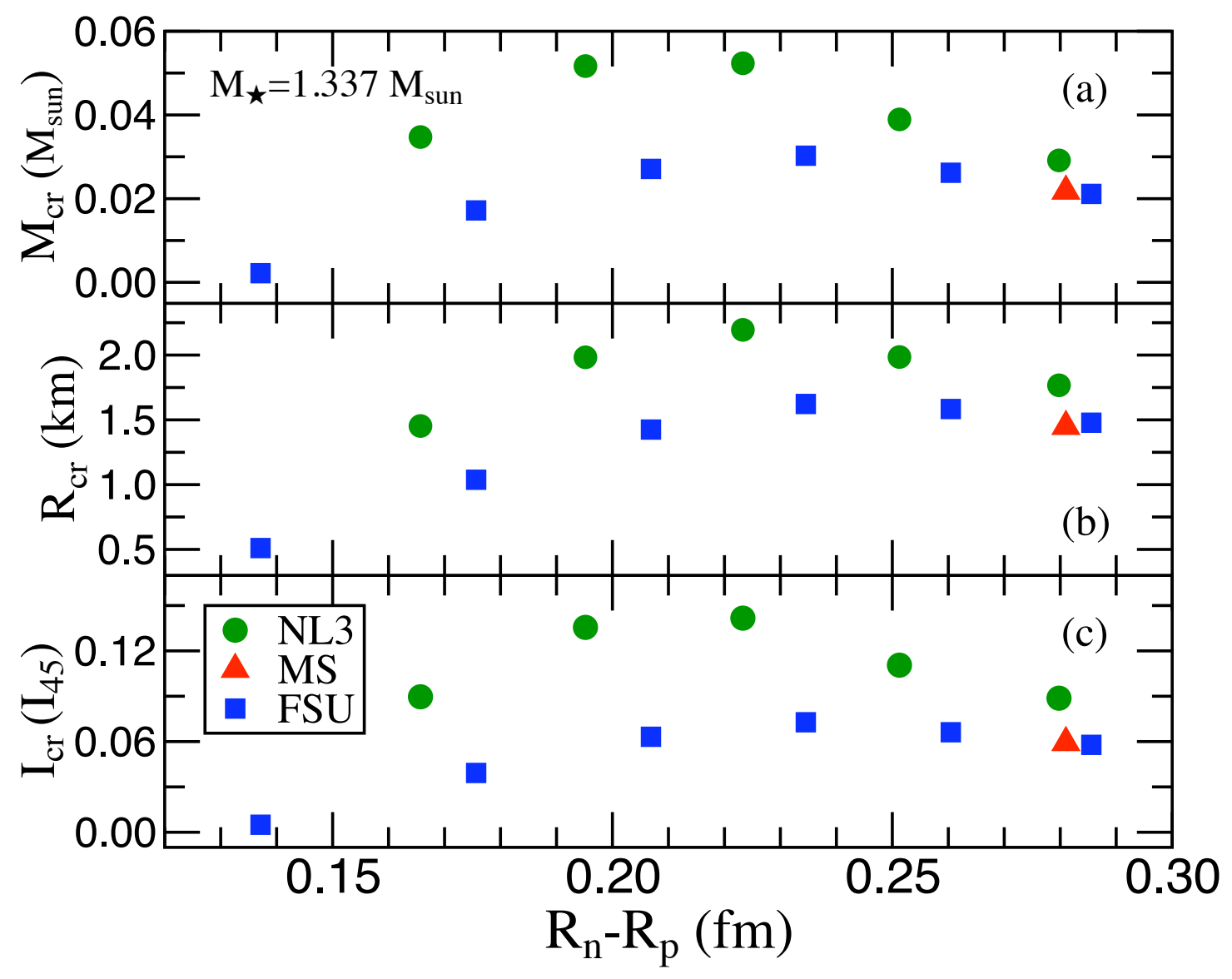

FIG. 7: (Color online) Crustal mass (a), crustal thickness (b), and crustal moment of inertia (c) as a function of the neutron skin thickness in ${ }^{208} \mathrm{~Pb}$ for a $1.337 M_{\odot}$ neutron star (J0737-3039 A). The various mean-field interactions are described in the text.

with such a soft symmetry energy may be in conflict with observation. Ultimately, such a low value for $I_{c r}$ can be traced back to the very small transition pressure predicted by the model. We close this section by mentioning that many of the results presented and discussed in graphical form have been collected in Tables IV] and V.

\section{CONCLUSIONS}

In the present contribution we have studied the sensitivity of the stellar moment of inertia to the underlying equation of state. In the slow-rotation approximation employed here, the stellar moment of inertia is only sensitive to the equation of state. Several equations of state were generated using relativistic mean-field models that have been accurately calibrated to the bulk properties of infinite nuclear matter and finite nuclei. As nuclear observables probe the EOS around nuclear matter saturation density, two aspects of the EOS remain poorly constrained even after the calibration procedure: (a) the density dependence of the symmetry energy and (b) the high-density component of the EOS. The relativistic mean-field models 


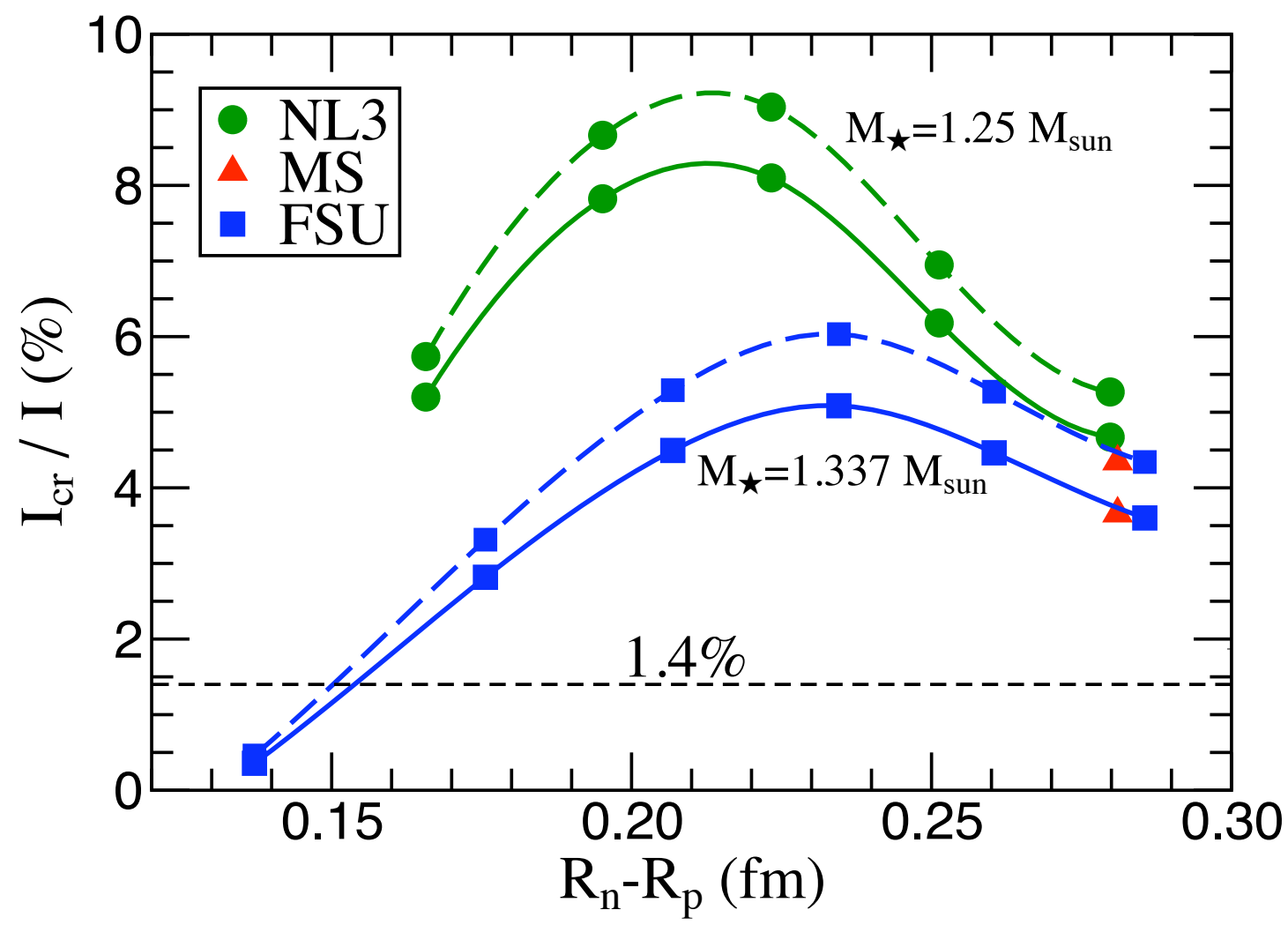

FIG. 8: (Color online) Fraction of the crustal moment of inertia as a function of the neutron skin thickness in ${ }^{208} \mathrm{~Pb}$ for the binary pulsar J0737-3039 with masses of $1.337 M_{\odot}$ (J0737-3039 A) and $1.25 M_{\odot}(\mathrm{J} 0737-3039 \mathrm{~B})$. The various mean-field interactions are described in the text.

employed here include two empirical parameters - $\Lambda_{\mathrm{v}}$ and $\zeta$ - that provide an efficient and economical way to modify the EOS. Whereas the former controls the density dependence of the symmetry energy, the latter controls the high density component of the EOS. As such, $\Lambda_{\mathrm{v}}$ can be used to tune the pressure of pure neutron matter at saturation density, thereby controlling the neutron radius of objects as diverse as finite nuclei and neutron stars. In contrast $\zeta$ - by modifying the high-density component of the EOS - strongly affects the maximum stellar mass that can be supported against gravitational collapse. By tuning these two parameters, one can generate limiting stellar masses that differ by more than one solar mass and radii (for a fixed stellar mass) that may differ by more than $2 \mathrm{~km}$. Note that one can generate these wide range of values without compromising the success of the models in reproducing a host of well determined nuclear observables.

With several equations of state in hand, we proceeded to compute the moment of inertia of the recently discovered binary pulsar PSR J0737-3039 (with individual masses of $M_{A}=$ $1.337 M_{\odot}$ and $\left.M_{B}=1.250 M_{\odot}\right)$. It has been suggested-due to the high sensitivity of the binary to general relativistic effects - that the moment of inertia of the fastest spinning pulsar in the binary (PSR J0737-3039A) may be measured with a 10\% accuracy. Our results indicate that knowledge of the pulsar moment of inertia (even with a $10 \%$ accuracy) could help discriminate among various equations of state. We note, however, that whereas our results suggests that a measurement of the moment of inertia could discriminate between equations of state that are either stiff or soft at high density, the sensitivity to the density 


\begin{tabular}{|l||c|c|c|c|c|c|c|c|c|}
\hline Model & $\Lambda_{\mathrm{v}}$ & $\rho_{c}$ & $R$ & $R_{\text {core }}$ & $M_{\text {core }}$ & $M_{\text {cr }} / M(\%)$ & $I$ & $I_{\text {core }}$ & $I_{\text {cr }} / I(\%)$ \\
\hline \hline NL3 & 0.00 & 4.672 & 15.14 & 13.30 & 1.307 & $2.2(2.1)$ & 1.901 & 1.811 & $4.7(4.7)$ \\
& 0.01 & 4.976 & 14.76 & 12.71 & 1.297 & $3.0(2.8)$ & 1.790 & 1.677 & $6.3(6.2)$ \\
& 0.02 & 5.030 & 14.58 & 12.33 & 1.284 & $4.0(3.8)$ & 1.751 & 1.607 & $8.2(8.1)$ \\
& 0.03 & 5.029 & 14.21 & 12.18 & 1.285 & $3.9(3.7)$ & 1.733 & 1.597 & $7.9(7.8)$ \\
& 0.04 & 5.017 & 13.68 & 12.18 & 1.302 & $2.6(2.6)$ & 1.725 & 1.635 & $5.2(5.3)$ \\
\hline MS & 0.00 & 7.136 & 13.92 & 12.45 & 1.315 & $1.6(1.6)$ & 1.620 & 1.560 & $3.7(3.7)$ \\
\hline FSU & 0.00 & 7.332 & 13.95 & 12.47 & 1.316 & $1.6(1.6)$ & 1.605 & 1.547 & $3.6(3.6)$ \\
& 0.01 & 8.316 & 13.49 & 11.85 & 1.311 & $2.0(1.9)$ & 1.482 & 1.415 & $4.5(4.5)$ \\
& 0.02 & 8.702 & 13.18 & 11.50 & 1.307 & $2.3(2.2)$ & 1.430 & 1.358 & $5.1(5.2)$ \\
& 0.03 & 8.855 & 12.77 & 11.35 & 1.310 & $2.0(2.0)$ & 1.406 & 1.343 & $4.5(4.6)$ \\
& 0.04 & 8.920 & 12.35 & 11.30 & 1.320 & $1.3(1.3)$ & 1.393 & 1.354 & $2.8(2.9)$ \\
& 0.05 & 8.937 & 11.84 & 11.33 & 1.335 & $0.2(0.2)$ & 1.387 & 1.382 & $0.4(0.4)$ \\
\hline
\end{tabular}

TABLE IV: Predictions for the properties of the J0737-3039 A pulsar with a mass of $M=1.337 M_{\odot}$ and spin period of $P=22.7 \mathrm{~ms}$ (or stellar frequency of $\Omega=276.8 \mathrm{~s}^{-1}[2]$ ). The central densities are in units of $10^{14} \mathrm{~g} / \mathrm{cm}^{3}$, the radii in $\mathrm{km}$, the core mass in solar masses and the moments of inertia in $10^{45} \mathrm{~g} \mathrm{~cm}^{2}$. The quantities in parenthesis are the analytic results for the fraction of the mass and moment of inertia contained in the solid crust (see text for details).

\begin{tabular}{|l||c|c|c|c|c|c|c|c|c|}
\hline Model & $\Lambda_{\mathrm{v}}$ & $\rho_{c}$ & $R$ & $R_{\text {core }}$ & $M_{\text {core }}$ & $M_{c r} / M(\%)$ & $I$ & $I_{\text {core }}$ & $I_{c r} / I(\%)$ \\
\hline \hline NL3 & 0.00 & 4.487 & 15.17 & 13.17 & 1.219 & $2.5(2.4)$ & 1.732 & 1.640 & $5.3(5.3)$ \\
& 0.01 & 4.804 & 14.79 & 12.56 & 1.208 & $3.4(3.2)$ & 1.624 & 1.508 & $7.1(7.0)$ \\
& 0.02 & 4.867 & 14.60 & 12.16 & 1.195 & $4.4(4.2)$ & 1.583 & 1.439 & $9.1(9.0)$ \\
& 0.03 & 4.872 & 14.19 & 11.99 & 1.196 & $4.3(4.1)$ & 1.564 & 1.428 & $8.7(8.6)$ \\
& 0.04 & 4.862 & 13.61 & 12.00 & 1.214 & $2.9(2.8)$ & 1.555 & 1.465 & $5.7(5.8)$ \\
\hline MS & 0.00 & 6.474 & 14.08 & 12.44 & 1.226 & $1.9(1.9)$ & 1.507 & 1.442 & $4.3(4.3)$ \\
\hline FSU & 0.00 & 6.634 & 14.13 & 12.48 & 1.226 & $1.9(1.9)$ & 1.497 & 1.434 & $4.2(4.2)$ \\
& 0.01 & 7.509 & 13.70 & 11.86 & 1.221 & $2.4(2.3)$ & 1.381 & 1.308 & $5.3(5.3)$ \\
& 0.02 & 7.871 & 13.36 & 11.49 & 1.216 & $2.7(2.6)$ & 1.329 & 1.249 & $6.0(6.0)$ \\
& 0.03 & 8.022 & 12.91 & 11.32 & 1.220 & $2.4(2.4)$ & 1.303 & 1.234 & $5.3(5.3)$ \\
& 0.04 & 8.091 & 12.44 & 11.27 & 1.231 & $1.5(1.5)$ & 1.288 & 1.246 & $3.3(3.3)$ \\
& 0.05 & 8.114 & 11.87 & 11.31 & 1.248 & $0.2(0.2)$ & 1.281 & 1.275 & $0.4(0.4)$ \\
\hline
\end{tabular}

TABLE V: Predictions for the properties of the J0737-3039 B pulsar with a mass of $M=1.250 M_{\odot}$ and spin period of $P=2.77 \mathrm{~ms}$ (or stellar frequency of $\Omega=2.268 \mathrm{~s}^{-1}[2]$ ). The central densities are in units of $10^{14} \mathrm{~g} / \mathrm{cm}^{3}$, the radii in $\mathrm{km}$, the core mass in solar masses and the moments of inertia in $10^{45} \mathrm{~g} \mathrm{~cm}^{2}$. The quantities in parenthesis are the analytic results for the fraction of the mass and moment of inertia contained in the solid crust (see text for details).

dependence of the symmetry energy appears to be weak - especially for models with a soft symmetry energy.

Although we find the total moment of inertia interesting, the main focus of this contribution was its crustal component $I_{c r}$. Several reasons prompted this choice. First, an analysis of pulsar glitches in the Vela pulsar suggests that at least $1.4 \%$ of the total moment of inertia must reside in the solid crust. This places significant constrains on the EOS. 
Second, the crust is thin and the density within it is low, so simple - yet fairly accurateanalytic expressions for $I_{c r}$ exist. These indicate that the crustal moment of inertia depends sensitively on a fundamental observable: the transition pressure at the core-crust interface $\left(P_{t}\right)$. Third, given the strong correlation between the core-crust transition density and the neutron skin thickness of ${ }^{208} \mathrm{~Pb}$, one expects a similar correlation to emerge in the case of $P_{t}$ and $I_{c r}$. Finally, given that at the time of this writing the Parity Radius Experiment (PREx) was actively collecting data, the prospects of constraining crustal properties with laboratory data appears imminent.

However, we found no correlation between the transition pressure $P_{t}$ and the neutron skin thickness in ${ }^{208} \mathrm{~Pb}$. Whereas a robust correlation exists between $R_{n}-R_{p}$ and various bulk properties of the EOS at the transition region - such as the baryon density, energy density, and proton fraction - no such correlation develops in the case of the transition pressure. And because of its sensitivity to the transition pressure, we conclude that the crustal moment of inertia will not be significantly constrained by a measurement of the neutron skin thickness in ${ }^{208} \mathrm{~Pb}$. This represents the main conclusion of our work. We found the explanation for this behavior subtle and rooted on the observed correlation between the transition density $\rho_{t}$ and $R_{n}-R_{p}$. As expected, the larger the value of $R_{n}-R_{p}$ the stiffer the symmetry energy. That is, given a fixed value of the baryon density the resulting pressure increases with $R_{n}-R_{p}$. However, the transition pressure is obtained by evaluating the EOS - not at a fixed value of the density, but rather - at the transition density, which is inversely correlated to $R_{n}-R_{p}$. It is precisely the fact that $\rho_{t}$ changes with $R_{n}-R_{p}$ that destroys any correlation between $P_{t}$ and $R_{n}-R_{p}$ [see Fig. 4(b)]. Although at odds with some studies that support the existence of such a correlation [35 38], our result appears consistent with a very recent analysis by Ducoin, Margueron, and Providencia [58].

\section{Acknowledgments}

This work was supported in part by a grant from the U.S. Department of Energy DEFD05-92ER40750.

\section{Appendix}

In this appendix we describe the necessary steps used to obtain the expression for the crustal moment of inertia given in Eq. (11). Given that the uniform liquid core accounts for most of the stellar mass and that the "fluid" in the crust behave non-relativistically, the following three approximations are assumed valid in the solid crust [23]:

$$
\begin{aligned}
& M(r) \approx M(R)=M, \\
& P(r) \ll \mathcal{E}(r), \\
& 4 \pi r^{3} P(r) \ll M(r)=M .
\end{aligned}
$$

Under these assumptions the equations for stellar structure simplify considerably. For example, Eq. (6) for the metric $\nu(r)$ reduces to

$$
\nu(r)=\frac{1}{2} \ln \left(1-\frac{R_{s}}{R}\right)-\frac{1}{2} R_{s} \int_{r}^{R} \frac{d x}{x^{2}-x R_{s}}=\frac{1}{2} \ln \left(1-\frac{R_{s}}{r}\right),
$$


where $R_{s} \equiv 2 G M$ is the Schwarzschild radius of the star. Similarly, in the crustal region the TOV-equation [Eq. (3)] becomes equal to

$$
\frac{d P(r)}{d r}=-\frac{G M \mathcal{E}(r)}{r^{2}\left(1-R_{s} / r\right)}=-\frac{R_{s} \mathcal{E}(r)}{2 r^{2}\left(1-R_{s} / r\right)}
$$

If not for the (important) general-relativistic correction $\left(1-R_{s} / r\right)^{-1}$, this expression would be identical to the equation of hydrostatic equilibrium in the purely Newtonian limit. Finally, the effective frequency $\bar{\omega}$ is approximated by its value at $r=R$. That is,

$$
\frac{\bar{\omega}(r)}{\Omega} \approx \frac{\bar{\omega}(R)}{\Omega}=\left[1-\left(\frac{R_{s}}{R}\right)\left(\frac{I}{M R^{2}}\right)\right]
$$

where we have made use of Eqs. (9b) and (10).

Using the above simplified expressions valid in the stellar crust, one obtains the following approximation for the crustal moment of inertia [22, 23, 34]:

$$
\begin{aligned}
I_{c r} & =\frac{8 \pi}{3}\left[1-\left(\frac{R_{s}}{R}\right)\left(\frac{I}{M R^{2}}\right)\right] \int_{R_{t}}^{R} r^{4} \frac{\mathcal{E}(r)}{1-R_{s} / r} d r \\
& =\frac{16 \pi}{3 R_{s}}\left[1-\left(\frac{R_{s}}{R}\right)\left(\frac{I}{M R^{2}}\right)\right] \int_{0}^{P_{t}} r^{6} d P .
\end{aligned}
$$

where the last line follows from using the simplified TOV equation [see Eq. (A.3)]. To perform the above integral we need an equation of state to compute the pressure profile $P=P(r)$ in the crust. As suggested in Refs. [22, 34], a polytropic equation of state of index $\gamma=4 / 3$ will be adopted for the crust. That is,

$$
P(\mathcal{E})=K \mathcal{E}^{\gamma}=K \mathcal{E}^{4 / 3},
$$

where $K$ is a constant.

Using such a simple - yet fairly accurate - EOS enables one to solve the TOV equation exactly. To do so, we first introduce the following scaling variables:

$$
x \equiv r / R_{s} ; \quad p \equiv P / P_{t} ; \quad \epsilon \equiv \mathcal{E} / \mathcal{E}_{t},
$$

where $P_{t} \equiv P\left(R_{t}\right)$ and $\mathcal{E}_{t} \equiv \mathcal{E}\left(R_{t}\right)$ are the pressure and energy density at the crust-core interface, with $R_{t}$ the transition (or core) radius. In terms of these scaling variables, the TOV-equation in the crust [Eq. A.3] ] takes the following simple form:

$$
\xi \frac{d p}{d x}=-\frac{p^{1 / \gamma}}{x(x-1)},
$$

where the small parameter $\xi \equiv 2 P_{t} / \mathcal{E}_{t}$ (of the order of a few percent) has been introduced. The above equation can now be integrated subject to the boundary condition $x=x_{t}=R_{t} / R_{s}$ at $p=1$. We obtain

$$
\begin{aligned}
x(p) & =\left(1-\left(1-x_{t}^{-1}\right) \exp \left[-\frac{\xi}{\alpha}\left(p^{\alpha}-1\right)\right]\right)^{-1} \\
& \approx x_{t}\left[1+\left(1-x_{t}\right)\left(p^{\alpha}-1\right) \frac{\xi}{\alpha}+\ldots\right]
\end{aligned}
$$


where $\alpha=(\gamma-1) / \gamma=1 / 4$ and the second line provides an approximation that is correct to first order in $\xi$. Although the integral appearing in Eq. A.5 can now be performed using standard numerical techniques, we prefer to provide an analytic expression for it by expanding the integrand in powers of the small parameter $\xi$. And although the results presented here are correct only to first-order in $\xi$, analytic expressions can be developed to arbitrary order. We obtain for an arbitrary power $n$

$$
\int_{0}^{1} x^{n}(p) d p=x_{t}^{n}\left[1+\frac{n}{1+\alpha}\left(x_{t}-1\right) \xi+\ldots\right]=x_{t}^{n}\left[1+\frac{4 n}{5}\left(x_{t}-1\right) \xi+\ldots\right] .
$$

Substituting the above expression into Eq. A.5 we obtain the following analytic expression for the crustal moment of inertia to first order in $P_{t} / \mathcal{E}_{t}$ :

$$
I_{c r} \approx \frac{16 \pi}{3} \frac{R_{t}^{6} P_{t}}{R_{s}}\left[1-\left(\frac{R_{s}}{R}\right)\left(\frac{I}{M R^{2}}\right)\right]\left[1+\frac{48}{5}\left(R_{t} / R_{s}-1\right)\left(P_{t} / \mathcal{E}_{t}\right)+\ldots\right] .
$$

Taking steps closely analogous to the ones followed for the crustal moment of inertia, the fraction of the stellar mass contained in the solid crust may be written as

$$
M_{c r}=4 \pi \int_{R_{t}}^{R} r^{2} \mathcal{E}(r) d r \approx 8 \pi R_{s}^{3} P_{t} \int_{0}^{1}\left(x^{4}(p)-x^{3}(p)\right) d p
$$

The integral can now be easily performed with the aid of Eq. (A.11). We obtain

$$
M_{c r} \approx 8 \pi R_{t}^{3} P_{t}\left(R_{t} / R_{s}-1\right)\left[1+\frac{32}{5}\left(R_{t} / R_{s}-3 / 4\right)\left(P_{t} / \mathcal{E}_{t}\right)+\ldots\right] .
$$


[1] M. Burgay et al., Nature. 426, 531 (2003).

[2] A. G. Lyne et al., Science 303, 1153 (2004).

[3] R. A. Hulse and J. H. Taylor, Astrophys. J. 195, L51 (1975).

[4] I. A. Morrison, T. W. Baumgarte, S. L. Shapiro, and V. R. Pandharipande, Astrophys. J. 617, L135 (2004).

[5] J. M. Lattimer and B. F. Schutz, Astrophys. J. 629, 979 (2005).

[6] M. Bejger, T. Bulik, and P. Haensel, Mon. Not. Roy. Astron. Soc. 364, 635 (2005).

[7] G. Lavagetto, I. Bombaci, A. D'Ai', I. Vidana, and N. R. Robba (2006), astro-ph/0612061.

[8] L. Iorio, New Astron. 14, 40 (2008).

[9] C. J. Horowitz and J. Piekarewicz, Phys. Rev. C64, 062802 (2001).

[10] C. J. Horowitz, S. J. Pollock, P. A. Souder, and R. Michaels, Phys. Rev. C63, 025501 (2001).

[11] K. Kumar, R. Michaels, P. A. Souder, and G. M. Urciuoli (2005), URL http://hallaweb. jlab.org/parity/prex.

[12] G. A. Baker, Phys. Rev. C60, 054311 (1999).

[13] H. Heiselberg, Phys. Rev. A63, 043606 (2002).

[14] J. Carlson, S.-Y. Chang, V. R. Pandharipande, and K. E. Schmidt, Phys. Rev. Lett. 91, 050401 (2003).

[15] A. Schwenk and C. J. Pethick, Phys. Rev. Lett. 95, 160401 (2005).

[16] Y. Nishida and D. T. Son, Phys. Rev. Lett. 97, 050403 (2006).

[17] K. Hebeler and A. Schwenk (2009), 0911.0483.

[18] S. Gandolfi, A. Y. Illarionov, S. Fantoni, F. Pederiva, and K. E. Schmidt, Phys. Rev. Lett. 101, 132501 (2008).

[19] A. Gezerlis and J. Carlson (2009), 0911.3907.

[20] J. Piekarewicz, J. Phys. G37, 064038 (2010), 0912.5103.

[21] I. Vidana, C. Providencia, A. Polls, and A. Rios, Phys. Rev. C80, 045806 (2009).

[22] B. Link, R. I. Epstein, and J. M. Lattimer, Phys. Rev. Lett. 83, 3362 (1999).

[23] J. M. Lattimer and M. Prakash, Phys. Rept. 442, 109 (2007).

[24] J. B. Hartle, Astrophys. J. 150, 1005 (1967).

[25] J. B. Hartle and K. S. Thorne, Astrophys. J. 153, 807 (1968).

[26] H. Mueller and B. D. Serot, Nucl. Phys. A606, 508 (1996).

[27] G. A. Lalazissis, J. Konig, and P. Ring, Phys. Rev. C55, 540 (1997).

[28] G. A. Lalazissis, S. Raman, and P. Ring, At. Data Nucl. Data Tables 71, 1 (1999).

[29] B. G. Todd-Rutel and J. Piekarewicz, Phys. Rev. Lett 95, 122501 (2005).

[30] J. B. Hartle, Astrophys. Space Sci. 24, 385 (1973).

[31] F. Weber, Pulsars as Astrophysical Laboratories for Nuclear and Particle Physics (Institute of Physics Publishing, Bristol, UK, 1999).

[32] N. K. Glendenning, Compact Stars (Springer-Verlag New York, 2000).

[33] L. D. Landau and E. M. Lifshitz, The Classical Theory of Fields (Pergamon Press, Oxford, 1975), 4th ed.

[34] J. M. Lattimer and M. Prakash, Astrophys. J. 550, 426 (2001).

[35] J. Xu, L.-W. Chen, B.-A. Li, and H.-R. Ma, Phys. Rev. C79, 035802 (2009).

[36] J. Xu, L.-W. Chen, B.-A. Li, and H.-R. Ma, Astrophys. J. 697, 1549 (2009).

[37] C. C. Moustakidis, T. Niksic, G. A. Lalazissis, D. Vretenar, and P. Ring (2010), 1004.3882. 
[38] A. Worley, P. G. Krastev, and B.-A. Li (2008), 0801.1653.

[39] C. P. Lorenz, D. G. Ravenhall, and C. J. Pethick, Phys. Rev. Lett. 70, 379 (1993).

[40] D. G. Ravenhall and C. J. Pethick, Astrophys. J. 424, 846 (1994).

[41] G. Baym, C. Pethick, and P. Sutherland, Astrophys. J. 170, 299 (1971).

[42] X. Roca-Maza and J. Piekarewicz, Phys. Rev. C78, 025807 (2008).

[43] D. G. Ravenhall, C. J. Pethick, and J. R. Wilson, Phys. Rev. Lett. 50, 2066 (1983).

[44] M. Hashimoto, H. Seki, and M. Yamada, Prog. Theor. Phys. 71, 320 (1984).

[45] C. J. Horowitz, M. A. Perez-Garcia, and J. Piekarewicz, Phys. Rev. C69, 045804 (2004).

[46] C. J. Horowitz, M. A. Perez-Garcia, J. Carriere, D. K. Berry, and J. Piekarewicz, Phys. Rev. C70, 065806 (2004).

[47] C. J. Horowitz, M. A. Perez-Garcia, D. K. Berry, and J. Piekarewicz, Phys. Rev. C72, 035801 (2005).

[48] J. Carriere, C. J. Horowitz, and J. Piekarewicz, Astrophys. J. 593, 463 (2003).

[49] J. D. Walecka, Annals Phys 83, 491 (1974).

[50] B. D. Serot and J. D. Walecka, Adv. Nucl. Phys. 16, 1 (1986).

[51] B. D. Serot and J. D. Walecka, Int. J. Mod. Phys. E6, 515 (1997).

[52] C. J. Horowitz and J. Piekarewicz, Phys. Rev. Lett. 86, 5647 (2001).

[53] B. G. Todd and J. Piekarewicz, Phys. Rev. C67, 044317 (2003).

[54] P. Danielewicz, R. Lacey, and W. G. Lynch, Science 298, 1592 (2002).

[55] J. Piekarewicz, Phys. Rev. C76, 064310 (2007).

[56] J. Piekarewicz, Phys. Rev. C69, 041301 (2004).

[57] R. J. Furnstahl, Nucl. Phys. A706, 85 (2002).

[58] C. Ducoin, J. Margueron, and C. Providencia (2010), 1004.5197. 